\title{
Author Correction: Association of a body shape index and hip index with cardiometabolic risk factors in children and adolescents: the CASPIAN-V study
}

\author{
Amir Kasaeian ${ }^{1,2} \cdot$ Zeinab Hemati $^{3} \cdot$ Ramin Heshmat $^{4} \cdot$ Fereshteh Baygi $^{5} \cdot$ Javad Heshmati $^{6}$. \\ Armita Mahdavi-Gorabi ${ }^{7} \cdot$ Mohammad Esmaeili Abdar $^{7} \cdot$ Mohammad Esmaeil Motlagh $^{8} \cdot$ Gita Shafiee $^{9}$. \\ Mostafa Qorban ${ }^{10,11} \cdot$ Roya Kelishadi $^{3}$
}

Published online: 2 April 2021

(C) Springer Nature Switzerland AG 2021

\section{Author Correction: J Diabetes Metab Disord. https://doi.org/10.1007/s40200-021-00743-0}

The 4th author name is Baygi.

The original article has been corrected.

Publisher's note Springer Nature remains neutral with regard to jurisdictional claims in published maps and institutional affiliations.

The online version of the original article can be found at https://doi.org/ 10.1007/s40200-021-00743-0

Mostafa Qorban

mqorbani1379@yahoo.com

Roya Kelishadi

roya.kelishadi@gmail.com

1 Hematology, Oncology and Stem Cell Transplantation Research Center, Research Institute for Oncology, Hematology and Cell Therapy, Tehran University of Medical Sciences, Tehran, Iran

2 Digestive Diseases Research Center, Digestive Diseases Research, Institute, Tehran University of Medical Sciences, Tehran, Iran

3 Child Growth and Development Research Center, Research Institute for Primordial Prevention of Non- Communicable Disease, Isfahan University of Medical Sciences, Isfahan, Iran

4 Chronic Diseases Research Center, Endocrinology and Metabolism Population Sciences Institute, Tehran University of Medical Sciences, Tehran, Iran

5 Center of Maritime Health and Society, Department of Public Health, University of Southern Denmark, Esbjerg, Denmark
6 Department of Nutritional Science, School of Nutritional Science and Food Technology, Kermanshah University of Medical Sciences, Kermanshah, Iran

7 Social Determinants of Health Research Center, Alborz University of Medical Sciences, Karaj, Iran

8 Pediatrics Department, Ahvaz Jundishapur University of Medical Sciences, Ahvaz, Iran

9 Endocrinology and Metabolism Research Center, Endocrinology and Metabolism Clinical Sciences Institute, Tehran University of Medical Sciences, Tehran, Iran

10 Non-communicable Diseases Research Center, Alborz University of Medical Sciences, Karaj, Iran

11 Department of Epidemiology, Chronic Diseases Research Center, Endocrinology and Metabolism Population Sciences Institute, Tehran University of Medical Sciences, Tehran, Iran 